\title{
Live monitoring onsite, remote and unattended data collection on synchrotron MX beamlines
}

\author{
D. Aragao, E. Nelson \\ Diamond Light Source, Harwell Science and Innovation Campus, Chilton, Didcot, OX11 ODE, UK \\ David.Aragao@diamond.ac.uk
}

Macromolecular crystallography instruments around the world run more and more in a remote access or unattended configuration. This leads to less contact between humans and the hardware as well as less awareness of software and hardware states. Beamline failures that were in the past routinely reported by humans are now missed and lost in the noise of other issues. On another hand there is a need to have a chain of triggers from the beamline failure to the call out of a synchrotron staff that can assess and fix an issue. Finally, although most facilities have constant monitoring tools such has text messages or emails on catastrophic failures like loss of vacuum or cooling in the DCM, they tend to not monitor less important values due to the incapacity of a human being to deal with excessive amounts of information including false positives. Here we present a beamline monitoring software that intents to monitor EPICS PVs as well as other systems via HTTP restful interfaces, database connections or on disk file analysis and report in a configurable way to systems such as Slack, Email, Signal/WhatsApp or others. The use of a Slack bot allows update of configuration notifications as well as query some beamline states remotely before a support remote connection is required. Concepts as beamline mode as well as custom notifications for different staff members as well as a dependency chain of failures help reduce the number of notifications to a level which can be dealt with. The expectation is that this will be part of the on call / callout system, monitor the beamline live for upcoming possible problems as well as provide a $\log$ of the beamline states for the last day(s).
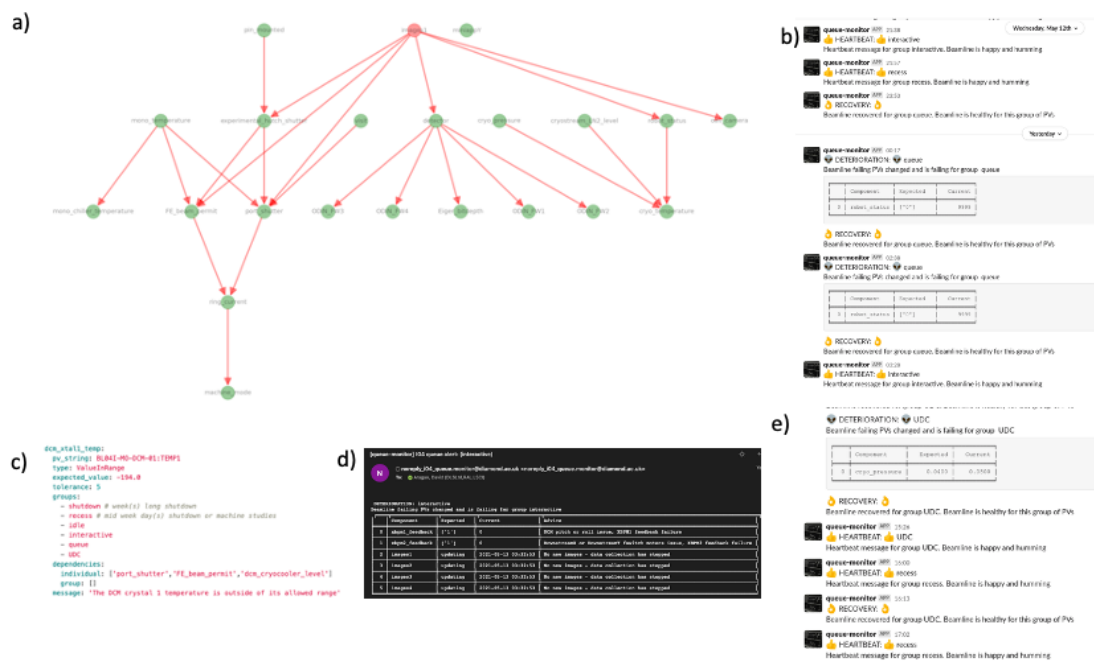

Figure 1. a) Live dependency diagram [1] for monitored EPICs PVs showing no images coming out of the but otherwise a healthy beamline. Notifications are only sent for PVs in failed state when no upstream dependency is not also in failed state. b \& e) Example slack notifications for beamline i04 [2] running with remote interactive users (c) and beamline i03 running unattended data collection. c) Example of the YAML configuration for monitoring DCM temperature with target, tolerance, beamline modes and dependency settings. d) Example email notification when a beam steering/feedback failure led to no data being collected by the detector.

\section{[1] https://networkx.org/}

[2] R. Flaig et all, Acta Cryst. (2017). A73, a71

Keywords: MX, beamline, monitor, call out, on call, remote, unattended, support, live, automation, resilience, pandemic, work from home

We thank the whole I04-1 beamline staff for lots of ideas taken from their queue-monitor script as well as the 2020 Diamond Light Source pandemic MX python focus group for suggestions both in code and ideas to solve issues that arose but in particular to Mark Williams and Louise Dunnett. 